\title{
On Dimension Reduction for the Power Control Problem
}

\author{
Luis Mendo and José M. Hernando
}

\begin{abstract}
In this letter, we show how the dimension of power control equation systems can be reduced from $K$, the number of users in the system, to $M$, the number of cells, without any loss of generality or accuracy. Decentralized downlink power control algorithms are then presented which generalize previously proposed ones broadening the range of application while maintaining reduced complexity.
\end{abstract}

Index Terms-Code-division multiple access, power control.

\section{INTRODUCTION}

$\mathbf{K}$ INDS of approaches to the power control problem can be found in the literature, namely "microscopic" descriptions in terms of the individual transmitted powers and "macroscopic" descriptions, where each base station is described by a variable that aggregates the effects of all users assigned to it. More specifically, for a cellular network with $M$ nonempty cells and $K$ active users, the microscopic description is equivalent to a $K$ th-order linear equation system in terms of the powers transmitted from (uplink) or toward (downlink) the mobile stations, whereas a macroscopic description consists of an $M$ th-order system in terms of a set of $M$ variables, one associated with each base station, from which the individual transmitted powers can be computed. The latter description is said to achieve dimension reduction with respect to the former, since the order of the equation system is reduced from $K$ to $M$.

The microscopic approach allows an accurate characterization of the system, whereas macroscopic descriptions have typically involved either some kind of approximation or loss of generality [1]-[3]. In this letter, we show that the exact microscopic description can be made completely equivalent to a macroscopic one, thus reducing complexity without compromising accuracy or generality.

As will be seen in Section IV, dimension reduction is directly applicable to practical power control algorithms in the downlink. In the uplink, dimension reduction can be exploited by means of centralized algorithms, but it does not appear to be applicable to decentralized power control algorithms, as discussed in Section V. In [1], a downlink decentralized power control algorithm is proposed which implicitly uses dimension reduction. Two versions of the algorithm are presented in the referenced paper, the second being an extension of the former to account for soft hand-off through macrodiversity combining. Both are restricted to the case where the desired signal level can be ap-

Paper approved by K. K. Leung, the Editor for Wireless Network Access and Performance of the IEEE Communications Society. Manuscript received January 12, 2000; revised May 19, 2000 and July 25, 2000.

The authors are with the Signals, Systems and Radiocommunications Department, Polytechnic University, 28040 Madrid, Spain.

Publisher Item Identifier S 0090-6778(01)01290-9. proximately included in the interference, all users have the same signal-to-interference ratio (SIR) target value and partial orthogonality can be neglected. The macrodiversity combining analysis (in which the individual SIRs add) further assumes a uniform distribution of SIR objectives among a given user's serving cells. The first two assumptions are not justified in third-generation systems, which support different service classes (which implies different SIRs), with the possibility of high bit rates (low processing gains). Based on the macroscopic description, we propose in Section IV downlink power control algorithms that generalize those in [1] so as to

- avoid regarding the desired signal as interference;

- allow unequal target SIRs;

- account for partial orthogonality;

- permit arbitrary SIR distribution among a given mobile's serving cells in the soft hand-off case

maintaining low complexity and decentralized operation.

The rest of the letter is organized as follows. In Section II we set up the system model and notation for the uplink, and show how the microscopic description can be reduced to a macroscopic one. Similar results are presented in Section III for the downlink. In Section IV, the generalized downlink power control algorithms are presented and their convergence is analyzed. Section V concludes with a discussion on the applicability of dimension reduction.

\section{UPLinK POWER CONTROL EQUATIONS}

We consider the uplink of a cellular code-division multipleaccess (CDMA) system with $M$ cell sites and $K$ users that are received by means of conventional matched-filter detection. A "snapshot" of the cellular network is analyzed. We assume that the assignment of mobile users to base stations is fixed, given by an assignment vector $\phi, \phi(k)$ being the base station that serves the $k$ th user. Note that this model includes the possibility of soft hand-off through macrodiversity selection, in which the serving base station for each mobile $k$ at a given instant is selected from a set of allowable cell sites. ${ }^{1}$ Propagation losses are described by an $M \times K$ attenuation matrix $\boldsymbol{\alpha}$, where $\alpha(m, k)$ is the path-loss from user $k$ to cell site $m$. The $M$-element noise vector $\mathbf{n}$ and the $K$-element target SIR vector $\gamma$ complete the system description. The former contains the thermal noise powers of all base stations, and the latter includes the target SIR values for each user before despreading (throughout this letter, "interference" should be interpreted as the sum of powers of the interfering signals plus thermal noise).

\footnotetext{
${ }^{1}$ Softer hand-off, based on macrodiversity combining, could be analyzed by a similar procedure to that in Section III-B.
} 
The condition that the SIR equals its target value for every user $k \in\{1, \ldots, K\}$

$$
\frac{\alpha(\phi(k), k) P(k)}{\sum_{\substack{j=1 \\ j \neq k}}^{K} \alpha(\phi(k), j) P(j)+n(\phi(k))}=\gamma(k)
$$

results in a linear system $\mathbf{A P}=\mathbf{N}$, where $\mathbf{A}$ is the $K \times K$ matrix given by

$$
A(i, j)= \begin{cases}-\alpha(\phi(i), j), & \text { for } i \neq j \\ \frac{\alpha(\phi(i), i)}{\gamma(i)}, & \text { for } i=j\end{cases}
$$

$\mathbf{N}=[n(\phi(1)) \cdots n(\phi(K))]^{\mathrm{T}}$ and $\mathbf{P}$ is the vector of unknown transmitted powers. This constitutes the microscopic description of the network. Assuming $\mathbf{A}$ nonsingular, the transmitted power values $P(k)$ can be computed from $\boldsymbol{\alpha}, \mathbf{n}, \boldsymbol{\gamma}$, and $\boldsymbol{\phi}$ in $O\left(K^{3}\right)$ operations as follows:

- $K$ divisions are needed for the diagonal elements of $\mathbf{A}$;

- $K^{3} / 3+K^{2}-K / 3$ multiplications/divisions (m/d) and $K^{3} / 3+K^{2} / 2-5 K / 6$ additions/subtractions (a/s) are required to solve the linear system by Gauss elimination [4, Theorem 3.5]

which amount a total of $K^{3} / 3+K^{2}+2 K / 3 \mathrm{~m} / \mathrm{d}$ and $K^{3} / 3+$ $K^{2} / 2-5 K / 6$ a/s.

In [2] a macroscopic description in terms of received powers is given for the case when all target SIRs are equal. Such description is possible because in this particular case the solution gives equal received powers for all the users assigned to the same base station. In [3] a different point of view is taken, in which an approximation is made to include the desired signal in the interference. A macroscopic $(M \times M)$ description is then derived in terms of certain interference variables (defined per base station). However, the approximation is not acceptable for relatively low processing gains. This undesirable feature can be avoided by means of a simple observation: the condition (1) for the SIR of a given user $k$ is equivalent to the following condition on the signal to signal-plus-interference ratio (SSIR):

$$
\frac{\alpha(\phi(k), k) P(k)}{\sum_{j=1}^{K} \alpha(\phi(k), j) P(j)+n(\phi(k))}=\frac{\gamma(k)}{1+\gamma(k)} \triangleq \tilde{\gamma}(k)
$$

This implies that the approximate description of [3] will be exact if the target values $\gamma(k)$ are substituted by $\tilde{\gamma}(k)$. Furthermore, a simpler formulation than that in [3] can be made if we work with the signal plus total interference instead of the internal interference as is done therein. For each base station $m$ let us define the variable $R(m)=\sum_{k=1}^{K} \alpha(m, k) P(k)+n(m)$, that represents the sum of all received power (including thermal noise) at base station $m$. The variables $R(m)$ verify the equation system

$$
\mathbf{G R}=\mathbf{n}
$$

with the $M \times M$ matrix $\mathbf{G}$ defined as

$$
G(m, n)=\delta_{m, n}-\sum_{k \in \phi^{-1}(n)} \frac{\alpha(m, k) \tilde{\gamma}(k)}{\alpha(n, k)} .
$$

From $\mathbf{R}$, the transmitted powers are readily calculated as

$$
P(k)=\frac{\tilde{\gamma}(k)}{\alpha(\phi(k), k)} R(\phi(k))
$$

This way, an exact calculation of the power values can be made in a general setting with $O\left(M^{3}+K M\right)$ operations: ${ }^{2}$

- $\mathbf{G}$ can be constructed from $\boldsymbol{\alpha}, \mathbf{n}, \boldsymbol{\gamma}$, and $\boldsymbol{\phi}$ with $2 M K \mathrm{~m} / \mathrm{d}$ and $M K$ a/s by the following procedure: initialize $\mathbf{G}$ to the identity matrix, and for every cell $m$ and every user $k$ subtract $(\alpha(m, k) / \alpha(\phi(k), k)) \tilde{\gamma}(k)$ from $G(m, \phi(k))$.

- The linear system (4) is solved with $M^{3} / 3+M^{2}-M / 3$ $\mathrm{m} / \mathrm{d}$ and $M^{3} / 3+M^{2} / 2-5 M / 6 \mathrm{a} / \mathrm{s}$.

- $\mathbf{P}$ is computed from $\mathbf{R}$ with $2 K \mathrm{~m} / \mathrm{d}$.

The total number of operations is thus $M^{3} / 3+M^{2}-M / 3+$ $2 K(M+1) \mathrm{m} / \mathrm{d}$ and $M^{3} / 3+M^{2} / 2+(K-5 / 6) M$ a/s.

In comparison with the microscopic description, and for the range of values of interest of $K$ and $M$, the macroscopic approach reduces the number of required operations.

\section{DOWNLINK POWER CONTROL EQUATIONS}

Dimension reduction can similarly be achieved for the downlink. Since soft hand-off is implemented in the downlink by diversity combining, the analysis is not as straightforward as in the uplink. An analysis without hand-off is firstly presented, and soft hand-off is then incorporated.

\section{A. Analysis Without Soft Hand-Off}

Assuming that every mobile is connected to only one cell, the downlink analysis is similar to that for the uplink, except for the following differences:

- the transmitter and receiver sides are interchanged;

- the base station transmits a pilot and other control (nontraffic) channels, which are seen as additional interference;

- interfering signals within the cell are partially orthogonal to the desired signal.

The system downlink is characterized by matrices $\alpha, \phi$, and $\gamma$ analogous to those of the uplink (with different values in general), a $K$-element noise vector $\mathbf{N}$, where $N(k)$ is the thermal noise power at mobile $k$, an $M$-element nontraffic power vector $\pi$, where $\pi(m)$ is the power transmitted by cell $m$ in the pilot and control channels, and an $M \times K$ orthogonality matrix $\rho$, where $\rho(m, k)$ is the orthogonality factor for signals from base station $m$ as seen by user $k$, i.e., if user $k$ is assigned to cell $m$, interference from the other signals transmitted by this cell is reduced by a factor $\rho(m, k)$.

Let us define $\beta(n, k, m)=\rho(m, k)$ if $m=n$ and 1 otherwise, and a vector $\overline{\mathbf{N}}$ with components $\bar{N}(k)=\sum_{n=1}^{M} \alpha(n, k) \beta(n, k, \phi(k)) \pi(n)+N(k)$. The microscopic system for the powers transmitted to the mobiles

\footnotetext{
${ }^{2} f(u, v)$ is said to be $O(g(u, v))$ if $f(u, v) \leq c g(u, v)$ for some positive constant $c$ and sufficiently high values of both $u$ and $v$ [5, Sec. 3.5].
} 
from their serving base stations is formulated as $\mathbf{A P}=\overline{\mathbf{N}}$, with

$$
A(i, j)= \begin{cases}-\alpha(\phi(j), i) \beta(\phi(j), i, \phi(i)), & \text { for } i \neq j \\ \frac{\alpha(\phi(i), i)}{\gamma(i)}, & \text { for } i=j\end{cases}
$$

Defining the vector $\mathbf{T}$, where $T(m)=\Sigma_{k \in \phi^{-1}(m)} P(k)+$ $\pi(m)$ is the total power transmitted by cell $m$, and

$$
\tilde{\gamma}(k)=\frac{\gamma(k)}{1+\rho(\phi(k), k) \gamma(k)}
$$

this linear system can be reduced to a macroscopic one

$$
\mathbf{H T}=\boldsymbol{\nu}
$$

and a set of $K$ equations

$P(k)=\frac{\tilde{\gamma}(k)}{\alpha(\phi(k), k)}\left(\sum_{n=1}^{M} \alpha(n, k) \beta(n, k, \phi(k)) T(n)+N(k)\right)$

with the matrix $\mathbf{H}$ and the column vector $\boldsymbol{\nu}$ given by

$$
\begin{aligned}
H(m, n) & =\delta_{m, n}-\sum_{k \in \phi^{-1}(m)} \frac{\alpha(n, k) \beta(n, k, m) \tilde{\gamma}(k)}{\alpha(m, k)}, \\
\nu(m) & =\pi(m)+\sum_{k \in \phi^{-1}(m)} \frac{\tilde{\gamma}(k) N(k)}{\alpha(m, k)} .
\end{aligned}
$$

The reduction in computational complexity is roughly similar to that in the uplink. Note that $\tilde{\gamma}(k)$ as given by (8) does not have the simple interpretation of a SSIR, because of the factor $\rho(\phi(k), k)$. It will nevertheless be referred to as SSIR in the sequel.

\section{B. Analysis with Soft Hand-Off}

A mobile in soft hand-off receives and combines signals transmitted to it by two or more cells. Assuming maximal-ratio combining, the resulting SIR is the sum of the SIRs corresponding to each of the received signals [6, Sec. 7.4].

A microscopic description can be made defining an $M \times K$ assignment matrix $\chi$, where $\chi(m, k)$ is the fraction of target SIR $\gamma(k)$ of mobile $k$ that is fulfilled by base station $m(\chi(m, k)=0$ if cell $m$ is not serving user $k$ ). Observe that this interpretation is possible due to the aforementioned additive decomposition of the SIR. ${ }^{3}$ The assignment matrix is a generalization of the assignment vector $\phi$ used in the analysis without hand-off (the latter being equivalent to $\left.\chi(m, k)=\delta_{m, \phi(k)}\right)$, and is assumed to be fixed. Imposing the condition $\sum_{m=1}^{M} \chi(m, k)=1$, the SIR experienced by user $k$ will be $\sum_{m=1}^{M} \chi(m, k) \gamma(k)=\gamma(k)$. Thus, each mobile can be "split" into $M$ fictitious mobiles with target SIRs $\chi(m, k) \gamma(m, k)$, and the situation is equivalent to an $M K$ user system without soft hand-off. Denoting the power transmitted by cell $m$ to user $k$ as $P(m, k)$, and defining $\gamma(m, k)=\chi(m, k) \gamma(k)$, a microscopic equation system can be written as in Section III-A. Also, defining $\tilde{\gamma}(m, k)=\gamma(m, k) /(1+\rho(m, k) \gamma(m, k))$ and

\footnotetext{
${ }^{3}$ It also requires that the target SIR for user $k$ be equal for all cells.
}

$T(m)=\sum_{k=1}^{K} P(m, k)+\pi(m)$ we obtain a macroscopic system

$$
\begin{aligned}
\mathbf{H T} & =\boldsymbol{\nu} \\
H(m, n) & =\delta_{m, n}-\sum_{k=1}^{K} \frac{\alpha(n, k) \beta(n, k, m) \tilde{\gamma}(m, k)}{\alpha(m, k)} \\
\nu(m) & =\pi(m)+\sum_{k=1}^{K} \frac{\tilde{\gamma}(m, k) N(k)}{\alpha(m, k)} \\
P(m, k) & =\frac{\tilde{\gamma}(m, k)}{\alpha(m, k)}\left(\sum_{n=1}^{M} \alpha(n, k) \beta(n, k, m) T(n)+N(k)\right)
\end{aligned}
$$

\section{Generalized Downlink POWER Control AlgORITHMS}

It has been shown that solving the macroscopic system by means of Gauss elimination reduces computational burden as compared to the microscopic one. However, this method of solution is centralized in nature. Iterative algorithms have been proposed [7]-[9], [1] that compute the transmitted powers in a distributed manner, and use a reduced number of measurements. These two aspects are of primary importance in practical applications.

In this section we provide extensions of the downlink Power Control and Diversity Power Control algorithms in [1]. Our algorithms generalize those in [1] in the directions stated in Section I, preserving decentralized operation and requiring only one additional measurement at the mobile per active base station. Note that the possibility to choose an arbitrary SIR distribution within the active set could be exploited to even the load between cells, i.e., to balance total transmitted powers.

\section{A. Generalized Power Control Algorithm}

Given $\boldsymbol{\gamma}, \boldsymbol{\phi}, \boldsymbol{\pi}$, a maximum power vector $\mathbf{T}_{\max }$ and an initial power vector $\mathbf{T}_{0}$, set $l=0$ and proceed as follows.

1) Allocating Individual Power Levels for Mobiles: For each cell $m$, obtain individual power levels $P_{l}(k)$ for $k \in \phi^{-1}(m)$ as

$$
\begin{aligned}
\psi_{l}(m, k) & =\frac{\tilde{\gamma}(k)}{\alpha(m, k)}\left(\sum_{n=1}^{M} \alpha(n, k) \beta(n, k, m) T_{l}(n)+N(k)\right) \\
\Psi_{l}(m) & =\sum_{k \in \phi^{-1}(m)} \psi_{l}(m, k) \\
P_{l}(k) & =\frac{\psi_{l}(m, k)}{\Psi_{l}(m)}\left(T_{l}(m)-\pi(m)\right) .
\end{aligned}
$$

2) Adjusting Cell-Site Transmit Power Levels: For each cell $m$, update cell-site power levels as

$$
T_{l+1}(m)=\min \left\{\Psi_{l}(m)+\pi(m), T_{\max }(m)\right\}
$$

set $l \leftarrow l+1$ and go to step 1).

The proposed algorithm consists, as that in [1], of two steps. Each cell allocates its total traffic power among its mobiles according to their individual needs. Then, if the target quality cannot be achieved, the total traffic power is adjusted, within the 
maximum power constraint. The generalization from the Power Control algorithm in [1] should be intuitively apparent taking into account that

- the interference from cell $n$ as seen by user $k$ assigned to cell $m$ is reduced by the factor $\beta(n, k, m)$ because of partial orthogonality;

- $\tilde{\gamma}(k)$ should replace $\gamma(k)$ to account for the fact that the desired signal, multiplied by $\rho(m, k)$, is being considered as part of the interference;

- users with different $\tilde{\gamma}(k)$ values should be allocated proportionally different powers. ${ }^{4}$

The algorithm can be given the following interpretation: step 1) can be seen as a SSIR balancing operation within each cell, for a fixed cell-site transmitted power, whereas step 2) essentially solves the macroscopic system (9) for the cell-site transmitted powers in an iterative manner, as we now show. Let us denote by $\tilde{\gamma}_{l}(k)$ the SSIR experienced at iteration $l$ by a given user $k$ assigned to cell $m$

$$
\tilde{\gamma}_{l}(k)=\frac{\alpha(m, k) P_{l}(k)}{\sum_{n=1}^{M} \alpha(n, k) \beta(n, k, m) T_{l}(n)+N(k)} .
$$

From (17) and (19), $\tilde{\gamma}_{l}(k)$ is computed as

$$
\tilde{\gamma}_{l}(k)=\frac{T_{l}(m)-\pi(m)}{\Psi_{l}(m)} \tilde{\gamma}(k)
$$

showing that the achieved SSIRs within a cell are proportional to their target values (i.e., every user achieves the same proportion of its desired SSIR). The relationship of step 2) of this algorithm (and that in [1] as a special case) with the macroscopic description (9) is obvious if we make use of (17), (18), (11), and (12) to write $\Psi_{l}(m)+\pi(m)=-\sum_{n=1}^{N} H(m, n) T_{l}(n)+\nu(m)+T_{l}(m)$. In view of this, the iteration in the step 2) of the algorithm can be interpreted, assuming that there is no power limitation due to $T_{\max }(m)$, as a matrix-separation iterative method [4, Sec. 9.6] to solve (9), in which the matrix $\mathbf{H}$ is separated into $\mathbf{I}_{M}$ and $\mathbf{H}-\mathbf{I}_{M}$, i.e., $\mathbf{T}_{l+1}=\left(\mathbf{I}_{M}-\mathbf{H}\right) \mathbf{T}_{l}+\boldsymbol{\nu}$.

The convergence of the Generalized Power Control algorithm can be easily analyzed within the framework of [9]. For convenience, let us denote this power control algorithm by $T_{l+1}(m)=$ $\mathcal{G}_{m}\left(\mathbf{T}_{l}\right)$. According to [9], in order to establish its convergence it is sufficient to assure that the algorithm is standard, i.e., satisfies the following properties for all $m$ and all $\mathbf{T}>\mathbf{0}$ (componentwise).

- Positivity: $\mathcal{G}_{m}(\mathbf{T})>0$.

- Monotonicity: If $\mathbf{T} \geq \mathbf{T}^{\prime}$, then $\mathcal{G}_{m}(\mathbf{T}) \geq \mathcal{G}_{m}\left(\mathbf{T}^{\prime}\right)$

- Scalability: For all $a>1, a \mathcal{G}_{m}(\mathbf{T})>\mathcal{G}_{m}(a \mathbf{T})$.

Proposition 1: The Generalized Power Control algorithm is standard.

\footnotetext{
${ }^{4}$ Observe that if all users have a common target $\tilde{\gamma}(k)=\tilde{\gamma}$ it can be factored out from the sum in (18), in accordance with [1].
}

Proof: The proof parallels that for the Power Control algorithm in [1]. For a given $\mathbf{T}$, the $m$ th component of the updated vector $\mathcal{G}_{m}(\mathbf{T})$ is obtained from (20) as

$$
\mathcal{G}_{m}(\mathbf{T})=\min \left\{\sum_{n=1}^{M} Z(m, n) T(n)+Y(m), T_{\max }(m)\right\}
$$

with

$$
\begin{aligned}
Z(m, n) & =\sum_{k \in \phi^{-1}(m)} \frac{\alpha(n, k) \beta(n, k, m) \tilde{\gamma}(k)}{\alpha(m, k)} \text { and } \\
Y(m) & =\sum_{k \in \phi^{-1}(m)} \frac{N(k) \tilde{\gamma}(k)}{\alpha(m, k)}+\pi(m) .
\end{aligned}
$$

The attenuations, target SSIRs, noise powers, and nontraffic powers are positive, and the orthogonality factors are nonnegative. This implies that $Z(m, n) \geq 0$ and $Y(m)>0$, which is sufficient for the positivity and monotonicity properties.

We now turn to scalability. Assuming $a>1$, if $\mathcal{G}_{m}(\mathbf{T})=$ $T_{\max }(m)$ we have

$$
a \mathcal{G}_{m}(\mathbf{T})=a T_{\max }(m)>T_{\max }(m) \geq \mathcal{G}_{m}(a \mathbf{T})
$$

and if $\mathcal{G}_{m}(\mathbf{T})<T_{\max }(m)$

$$
\begin{aligned}
\mathcal{G}_{m}(a \mathbf{T}) & =\min \left\{a \sum_{n=1}^{M} Z(m, n) T(n)+Y(m), T_{\max }(m)\right\} \\
& \leq a \sum_{n=1}^{M} Z(m, n) T(n)+Y(m) \\
& =a \mathcal{G}_{m}(\mathbf{T})+(1-a) Y(m)<a \mathcal{G}_{m}(\mathbf{T}) .
\end{aligned}
$$

As a consequence of Proposition 1, if there is a solution $\mathbf{T}$ with $\mathbf{0}<\mathbf{T} \leq \mathbf{T}_{\max }$, the algorithm converges to it, i.e., $T_{l}(m) \rightarrow T(m)$ and $\Psi_{l}(m) \rightarrow T(m)-\pi(m)$ as $l \rightarrow \infty$.

We now investigate the convergence of the experienced SIRs to their target values. Let $\gamma_{l}(k)$ denote the SIR experienced by a mobile $k \in \phi^{-1}(m)$ at the $l$ th iteration

$$
=\frac{\alpha(m, k) P_{l}(k)}{\sum_{n=1}^{M} \alpha(n, k) \beta(n, k, m) T_{l}(n)-\alpha(m, k) \rho(m, k) P_{l}(k)+N(k)}
$$

Using (22) and the identity $\gamma_{l}(k)=\tilde{\gamma}_{l}(k) /\left(1-\rho(m, k) \tilde{\gamma}_{l}(k)\right)$, and then substituting $\tilde{\gamma}(k)$ for $\gamma(k) /(1+\rho(m, k) \gamma(k))$ gives

$$
\gamma_{l}(k)=\frac{\left(T_{l}(m)-\pi(m)\right) \gamma(k)}{\Psi_{l}(m)+\left[\Psi_{l}(m)-\left(T_{l}(m)-\pi(m)\right)\right] \rho(m, k) \gamma(k)} .
$$

Since $T_{l}(m) \rightarrow T(m)$ and $\Psi_{l}(m) \rightarrow T(m)-\pi(m)$ as $l \rightarrow \infty$, it is clear that $\tilde{\gamma}_{l}(k) \rightarrow \tilde{\gamma}(k)$ and $\gamma_{l}(k) \rightarrow \gamma(k)$ as $l \rightarrow \infty$.

We have already observed from (22) that the experienced SSIRs $\tilde{\gamma}_{l}(k)$ within each cell $m$ are balanced at each iteration step. The SIRs $\gamma_{l}(k)$, however, are not proportional to their target values for finite $l$, due to the second term in the denominator of (24). Nonetheless, as $T_{l}(m)$ and $\Psi_{l}(m)$ approach their convergence values, the mentioned term tends to 0 , so that the 
imbalance vanishes. This is coherent with the fact that $\gamma_{l}(k) \rightarrow$ $\gamma(k)$ as $l \rightarrow \infty$. Moreover, with the Generalized Power Control algorithm, the experienced SIR $\gamma_{l}(k)$ is always closer to $\gamma(k)$ than that obtained using the Power Control algorithm in [1], as we now show. We set $\rho(n, k)=1$ and $\gamma(k)=\gamma$ $\forall n \in\{1, \ldots, M\}, k \in\{1, \ldots, K\}$, which is the most favorable case for the latter. Let $k$ denote an arbitrary user, and let $m=\phi(k)$. It should be observed that in the discussion on balancing link quality in [1, Sec. III-A] the experienced SSIRs, not the SIRs, are actually computed. Bearing this in mind, [1, eq. (10)] is rewritten in our notation as

$$
\tilde{\gamma}_{l}(k)=\frac{\alpha(m, k) P_{l}(m)}{\sum_{n=1}^{M} \alpha(n, k) T_{l}(n)+N(k)}=\frac{T_{l}(m)-\pi(m)}{\Psi_{l}(m)} \gamma
$$

The corresponding SIR is

$$
\gamma_{l}(k)=\frac{\tilde{\gamma}_{l}(k)}{1-\tilde{\gamma}_{l}(k)}=\frac{T_{l}(m)-\pi(m)}{\Psi_{l}(m)-\left(T_{l}(m)-\pi(m)\right) \gamma} \gamma .
$$

We see that the SIRs are not actually balanced with the Power Control algorithm either. Furthermore, since $T_{l}(m)-\pi(m)$ and $\Psi_{l}(m)$ tend to the same value, according to $(26) \gamma_{l}(k)$ does not converge to $\gamma$, but to a slightly different value $\gamma /(1-\gamma)$ (this could have been expected, since in that algorithm the target SIRs are treated as SSIRs). On the contrary, the Generalized Power Control algorithm gives, particularizing (24)

$$
\gamma_{l}(k)=\frac{T_{l}(m)-\pi(m)}{\Psi_{l}(m)+\left[\Psi_{l}(m)-\left(T_{l}(m)-\pi(m)\right)\right] \gamma} \gamma
$$

Comparing (26) and (27), we see that the additional $\Psi_{l}(m)$ in the latter tends to cancel $T_{l}(m)-\pi(m)$, moving $\gamma_{l}(k)$ closer to $\gamma$ than in (26) (and arbitrarily close as $l \rightarrow \infty$ ).

In practical systems the values $\tilde{\gamma}(k)$ and $\psi(m, k)$ required in the algorithm can be obtained in the following way. Each mobile $k$, with $\phi(k)=m$, measures $\sum_{n=1}^{M} \alpha(n, k) T(n)+N(k)$ and $\sum_{n=1}^{M} \alpha(n, k) \beta(n, k, m) T(n)+N(k)$. The former is simply the total received power, and the latter can be obtained by correlating the received signal with the expected received waveform 5 corresponding to an unused ("dummy") downlink channel in cell $m .^{6}$ Subtracting both measurements $\alpha(m, k)(1-\rho(m, k)) T(m)$ is obtained, and $\rho(m, k)$ can be readily calculated using knowledge of $T(m)$ and assuming that the attenuation $\alpha(m, k)$ can be estimated. With the values $\sum_{n=1}^{M} \alpha(n, k) \beta(n, k, m) T(n)+N(k), \rho(m, k)$ and $\alpha(m, k)$ of all its mobiles, the base station is able to compute $\tilde{\gamma}(k)$ and $\psi(m, k)$.

\section{B. Generalized Diversity Power Control Algorithm}

Given $\boldsymbol{\gamma}, \boldsymbol{\chi}, \boldsymbol{\pi}, \mathbf{T}_{\max }$, and $\mathbf{T}_{0}$, set $l=0$ and proceed as follows.

${ }^{5}$ That is, a weighted sum of delayed replicas of the corresponding code sequence, as used in the Rake receiver.

${ }^{6}$ For a given multipath profile, the orthogonality factor depends on the chip rate but not on the bit rate. For a system with multiple bit rates (achieved by variable spreading-factor orthogonal codes [10]), the correlation interval is necessarily one modulation period of the "dummy" channel (or an integer multiple thereof), and the result must be properly scaled to compare with $\sum_{n=1}^{M} \alpha(n, k) T(n)+N(k)$.
1) Allocating Individual Power Levels for Mobiles: For each cell $m$, obtain individual power levels $P_{l}(m, k)$ for $k$ with $\chi(m, k) \neq 0$ as

$\psi_{l}(m, k)=\frac{\tilde{\gamma}(m, k)}{\alpha(m, k)}\left(\sum_{n=1}^{M} \alpha(n, k) \beta(n, k, m) T_{l}(n)+N(k)\right)$

$\Psi_{l}(m)=\sum_{k=1}^{K} \psi_{l}(m, k)=\sum_{k: \chi(m, k) \neq 0} \psi_{l}(m, k)$

$P_{l}(m, k)=\frac{\psi_{l}(m, k)}{\Psi_{l}(m)}\left(T_{l}(m)-\pi(m)\right)$.

2) Adjusting Cell-Site Transmit Power Levels: For each cell $m$, update cell-site power levels as

$$
T_{l+1}(m)=\min \left\{\Psi_{l}(m)+\pi(m), T_{\max }(m)\right\}
$$

set $l \leftarrow l+1$ and go to step 1$)$.

Proposition 2: The Generalized Diversity Power Control algorithm is standard.

Proof: This algorithm is equivalent to the Generalized Power Control algorithm applied to $M K$ users, where the $(m, k)$ th user is assigned to cell $m$ with target SIR $\gamma(m, k)=\gamma(k) \chi(m, k)$, orthogonality factor $\rho(m, k)$, attenuations $\alpha(1, k), \ldots, \alpha(M, k)$ and noise power $N(k)$.

The values $\tilde{\gamma}(m, k)$ and $\psi(m, k)$ can be obtained as before, except that each mobile $k$ now measures $\sum_{n=1}^{M} \alpha(n, k) T(n)+$ $N(k)$ and a different $\sum_{n=1}^{M} \alpha(n, k) \beta(n, k, m) T(n)+N(k)$ for each active cell $m$, and the corresponding values $\rho(m, k)$ must be computed.

\section{APPliCABILITY OF THE MACROSCOPIC DESCRIPTION}

We have shown that an exact macroscopic description for the power control problem in CDMA cellular networks can be formulated, reducing computational complexity from $O\left(K^{3}\right)$ to $O\left(M^{3}+M K\right)$. The (centralized) direct method of solution of the macroscopic equations can be used when information about the system parameters is available in a centralized manner, such as in simulation analyses.

Dimension reduction is also applicable in decentralized implementations of power control algorithms in the downlink case. We have proposed decentralized power control algorithms that iteratively solve the macroscopic equation systems. These algorithms improve the accuracy and extend the field of application of those presented in [1].

Regarding uplink decentralized power control algorithms, dimension reduction does not seem to be applicable in this case, because the macroscopic variables $R(m)$ cannot be directly controlled, but only by means of the (microscopic) transmitted powers.

\section{ACKNOWLEDGMENT}

The quality of the letter has been improved from the valuable comments made by the reviewers. 


\section{REFERENCES}

[1] D. Kim, "A simple algorithm for adjusting cell-site transmitter power in CDMA cellular systems," IEEE Trans. Veh. Technol., vol. 48, pp. 1092-1098, July 1999.

[2] S. V. Hanly, "Information Capacity of Radio Networks," Ph.D. dissertation, Cambridge Univ., Cambridge, U.K., Aug. 1993.

[3] - "Congestion measures in DS-CDMA networks," IEEE Trans. Commun., vol. 47, pp. 426-437, Mar. 1999.

[4] B. Noble and J. W. Daniel, Applied Linear Algebra, 3rd ed. Englewood Cliffs, NJ: Prentice-Hall, 1988.

[5] G. Brassard and P. Bratley, Fundamentals of Algorithmics. Englewood Cliffs, NJ: Prentice-Hall, 1996.
[6] J. G. Proakis, Digital Communications, 2nd ed. New York: McGrawHill, 1989.

[7] S. V. Hanly, "An algorithm for combined cell-site selection and power control to maximize cellular spread spectrum capacity," IEEE J. Select. Areas Commun., vol. 13, pp. 1332-1340, Sept. 1995.

[8] R. D. Yates and C.-Y. Huang, "Integrated power control and base station assignment," IEEE Trans. Veh. Technol., vol. 44, pp. 638-644, Aug. 1995.

[9] R. D. Yates, "A framework for uplink power control in cellular radio systems," IEEE J. Select. Areas Commun., vol. 13, pp. 1341-1347, Sept. 1995.

[10] E. H. Dinan and B. Jabbari, "Spreading codes for direct sequence CDMA and wideband CDMA cellular networks," IEEE Commun. Mag., pp. 48-54, Sept. 1998. 